\title{
How to carve up the world: Learning and collaboration for structure recommendation
}

\author{
Mathias Verbeke, Ilija Subašić, and Bettina Berendt \\ Department of Computer Science, KU Leuven, Belgium \\ \{mathias.verbeke, bettina. berendt\}@cs.kuleuven. be \\ subasic.ilija@gmail.com
}

\begin{abstract}
Structuring is one of the fundamental activities needed to understand data. Human structuring activity lies behind many of the datasets found on the internet that contain grouped instances, such as file or email folders, tags and bookmarks, ontologies and linked data. Understanding the dynamics of large-scale structuring activities is a key prerequisite for theories of individual behaviour in collaborative settings as well as for applications such as recommender systems. One central question is to what extent the "structurer" - be it human or machine - is driven by his/its own prior structures, and to what extent by the structures created by others such as one's communities.

In this paper, we propose a method for identifying these dynamics. The method relies on dynamic conceptual clustering, and it simulates an intellectual structuring process operating over an extended period of time. The development of a grouping of dynamically changing items follows a dynamically changing and collectively determined "guiding grouping". The analysis of a real-life dataset of a platform for literature management suggests that even in such a typical "Web 2.0" environment, users are guided somewhat more by their own previous behaviour than by their peers. Furthermore, we also illustrate how the presented method can be used to recommend structure to the user.
\end{abstract}

Keywords: Collaborative classification, social web mining, user modelling, structure recommendation, divergence measure

\section{Introduction}

Nowadays, people are faced with a huge amount of data, originating from the increasing amount of emails, stored documents, or scientific publications. Just as people order their CD collection according to genre, sort books on shelves by author, or clothes by colour, it lies in the human nature to structure digital items. This is supported by the directory structure on desktop computers or tags for bookmarks, photos, and online publications. The structuring of data is a diverse process, and the grouping of a set of items into subsets can be seen as investing it with semantics: a structuring into sets of items, each instantiating a concept.

In addition, these concepts depend on each other: One concept is what the other is not; there is meaning not only in assigning an item to a concept, but also 
in not assigning it to another concept; and the abstract concepts co-evolve with the assignment of concrete instances to them. This feature makes an analysis of grouping more complex than an analysis just focusing on "bipartite" relations between items and concepts, on the growth of concept vocabulary over time, etc.

Since the end result of grouping will depend on the context, tasks, and previous knowledge of the "structurer", there is no overall "optimal" grouping. Grouping according to needs and knowledge is a prerequisite for the grouping to make sense to the user. Despite the personal nature of this process, people tend to be influenced by others - be it their real-life social circle, their online friends, or social recommender systems, which could steer the user (and the grouping) in a certain direction.

A good understanding of the dynamics of these structuring activities is a key prerequisite for theories of individual behaviour in collaborative settings, and it is necessary to improve the design of current and next generation (social) recommender systems $[1,15]$. Furthermore, it can leverage the design of mechanisms that rely on implicit user interactions such as social search $[10,8]$. The goal of this paper is to develop a dynamic conceptual clustering [20] that simulates this intellectual structuring process which is able to identify these structuring dynamics. The evolution of the grouping of an individual user is influenced by dynamically changing and collectively determined "guiding grouping(s)", which we will refer to as guides. In this paper, we investigated two types of guides. The first one is motivated by a "narrow" view of the structurer's prior grouping behaviour and experience, while the second one starts from a "wider" perspective that also takes peer experience into account in grouping decisions.

The process in which we want to transfer the structuring of one set of items to another set of items is a combination of two data mining tasks. First, we learn a model for this grouping (classification), which can be applied to structure alternative sets of items. We refer to this model as the grouping's intension, as opposed to its extension, which is the original, un-annotated grouping of the items. The first task starts with an extension of a structuring, and learns its intension as a classification model. The second task is to use the intensions of the peer groupings and apply their classifiers for prediction, to structure a new item. It starts from defining the $\mathrm{k}$ nearest peer groupings for a user. To decide on the $\mathrm{k}$ nearest peer groupings in a situation where peers group different items, we defined a novel measure of divergence between groupings that may have a different number and identities of items. Once we obtain the $\mathrm{k}$ nearest peers, the task is to decide on item-to-group assignment using their groupings. Based on the presence of an item in peer groupings, this decision is based either on the extension of the peer grouping (when a peer already grouped the item) or on its intension (when a peer has not grouped the item yet). By comparing the possible end groupings with the actual end grouping, we see which guide is more likely to have determined the actual process.

Our main contributions are: (a) a new data-mining approach that learns an intensional model of user groupings and uses these to group new items. The method can be used to identify structuring dynamics, which simulates an in- 
tellectual structuring process operating over an extended period of time, (b) a new divergence measure to define divergence between groupings of non-identical item sets, (c) a study of grouping behaviour in a social bookmarking system, and (d) two systems illustrating the proposed method to recommend structure to the user.

This paper is structured as follows. The following section reviews related work. Section 3 describes the method for identifying the structuring dynamics via grouping guidance, where we first outline the general system workflow and subsequently present a formalisation of the different parts. Section 4 contains an empirical analysis of this process that centres on item grouping in a real-life data set. In Section 5 we show that the grouping of new objects can also be used to recommend structure to a user, which is illustrated by means of two systems embodying this idea. Finally, Section 6 concludes the paper and presents an outlook for future work.

\section{Related work}

Our work is closely related to the research on personalised information organisation, where the goal is to understand how the user can be supported in searching, exploring, and organising digital collections. This field studies how users structure data collections. Current approaches usually concentrate on individual aspects such as search (e.g. personalised ranking), rudimentary exploration support and visualisation. In [18] the authors present a taxonomy of the features that can be used for this purpose. They introduce content-based features (e.g., word distributions), content-descriptive features (e.g., keywords or tags describing textual or image content), and context-independent metadata (e.g., creator) features. The key questions from this domain that are relevant to the topic of this paper are: How is it possible to support a user in grouping collections of items?, How can we use information about the way a user groups collections to support him in structuring as-yet-unknown collections? Nürnberger and Stober [23] give an answer to this question. They describe an adaptive clustering approach that adapts to the user's way of structuring, based on a growing self-organising map. As illustrated in [3], hierarchical clustering can help to structure unseen collections based on the way a user structures her own collections. It models the structuring criteria of a user by constraints in order to learn feature weights. The goal of our approach is to examine these structuring dynamics and investigate how a user is guided by himself or his peers. This can be very useful in detecting a user's guiding preferences and obtaining an overview of the user characteristics in the overall system.

In characterisation studies, system usage patterns are studied to propose models which explain and predict user behaviour in these systems. A number of studies have investigated tagging systems. Closest to our work is the research by Santos-Neto et al. [26] on individual and social behaviour in tagging systems. They define interest-sharing between users as a measure for inferring an implicit social structure for the tagging community. In addition, they investigate whether a high level of tag reuse results in users that tag overlapping sets of items and/or 
use overlapping sets of tags. Sen et al. [28] study how a tagging community's vocabulary of tags forms the basis for social navigation and shared expression. They present a user-centric model of vocabulary evolution in tagging communities based on community influence and personal tendency, with an evaluation on a real system, namely the MovieLens recommender system. Musto et al. [22] investigate the relation between tagging based on one's own past tags or on the community's tags, abstracting from the dynamics of tag assignments.

However, the studies of tagging are limited to a "bipartite" view of the binary relations between tags and items (or the ternary ones between tags, items and users). They do not take into account the relations between tag assignments and tag non-assignments, i.e. the way in which different concepts interact structurally, for example by being in competition with each other.

Our method complements these results with insights into structuring dynamics and behavioural characteristics in collaborative settings. We focus on the way users group items and how they are influenced during this process. This can help to improve the design of future collaborative systems and leverage the design of mechanisms that rely on implicit user interactions.

From conceptual and predictive clustering [6,34], we used the key idea to a) form clusters of elements, then b) learn classifiers that reconstruct these clusters and c) apply the classifier for further result sets. Research on ontology re-use, in particular adaptive ontology re-use [30], investigates the modelling and mapping steps needed for re-using given ontologies, for whatever purpose. In contrast, we concentrate on re-use for grouping/classifying new objects, and on how to find the most suitable ontologies for re-use.

\section{Grouping guidance}

This section introduces notation (Section 3.1) and describes the system's general workflow and the individual steps.

The system observes, for each user, an initial grouping and learns an initial classifier from it (Section 3.2). It then identifies, for a given user, which classifier to use next - the user's own or one determined by his peers (Section 3.3). It applies the chosen classifier (Section 3.4) and then updates it to reflect the new item grouping, which now also contains the just-added item (Section 3.5).

Steps 2, 3 and 4 are iterated on any item that the users want to structure. To analyse the structuring behaviour of each user, we compare the resulting groupings of this process to the real user groupings, which are the result of the user' structuring without computational assistance.

In Section 3.3, we present our measure of divergence between user groupings of non-identical item sets.

\subsection{Notation}

In this section we will introduce notational conventions for the basic concepts, for the groupings of each user's items and the classifiers learned from them, and for the time points through which the structuring evolves. Specific instantiations of these concepts can be found in Figures 1 and 2. 
Basic components We use the following notation:

- Let $U$ denote the set of all users (used symbols: $u, v, w$ ).

- Let $T$ denote the set of all time points $\left\{0,1, \ldots, t_{\max }\right\}$, where $t_{\max }$ represents the time at which the last item arrives.

- Let $D$ denote the set of all items (used symbol: $d$ ).

D will be used in combination with subscripts that represent users. Its superscripts represent time points. Thus $D_{u}^{t} \subseteq D$ denotes the set of all $d \in D$ already considered by $u \in U$ at $t \in T$. The item assigned to the structure by user $u$ at $t$ is an addition to this; it is represented by $d_{u}^{t} \in\left(D \backslash D_{u}^{t}\right)$.

Groupings and classifiers $G$ and $C$ are the (machine-induced) groupings for each user's items and the classifiers learned from them, respectively. $G$ can be any of following:

$O G$ - Observed Grouping This represents the grouping of the user at the start of the learning phase. As will be clarified in subsection 3.2, this will be the grouping used to learn the initial classifier at the start of the cycle.

$G S$ - Simulated Grouping, guided by self This represents the grouping, at a certain point in time during the cycle, that is solely generated by the classifier of the user in question. This will be specified in subsection 3.4.

$G n$ - Simulated Grouping, guided by $n$ peers Just as $G S$, this represents the grouping at a certain point in time during the cycle, but now the grouping is guided by $n$ peers of the user under consideration. This will be specified in subsection 3.4.

As was the case for item sets, subscripts denote users and superscripts denote time points. $G_{u}^{t}$ is the grouping that holds at time $t$ for user $u$ (the set of $u$ 's item sets), i.e. a function that partitions the user's current items into groups: $G_{u}^{t}: D_{u}^{t} \mapsto 2^{D}$. We will refer to this as the extensional definition of a concept, where the definition is formed by listing all objects that fall under that definition, and thus belong to the set or class for which the definition needs to be formed.

$C_{u}^{t}$ is the classifier (intension) learned from $G_{u}^{t}$, modelled as the function specifying an item's group according to this classifier: $C_{u}^{t}(d)=x$ with $C_{u}^{t}$ : $D \mapsto 2^{D}$. The intensional definition is a concept that originated from logic and mathematics. In this context, an intensional definition is defined as a set of conditions for an object to belong to a certain class. This is done by specifying all the common properties of the objects that are part of this set, with the goal of capturing its meaning. Analogously to $O G, G S$ and $G n$ for groupings, $O C$, $C S$ and $C n$ are defined as the initial classifier and the classifiers guided by "self" and by $n$ peers, respectively.

Time Structuring is modelled as evolving through a series of discrete time points, where each new structuring-related activity (such as tagging a document in a social-bookmarking system) happens at some time point and leads to either 
the assimilation of the item into the given conceptual structure of $G$ and $C$, or to a modification of the conceptual structure to accommodate the item. The conceptual structure remains stable until the subsequent activity.

We will refer to next $(t, u)$ with next $: T \times U \mapsto T$ as the first time point after $t$ at which $u$ structures an item, which changes the item set to $D_{u}^{n e x t(t, u)}=D_{u}^{t} \cup$ $\left\{d_{u}^{t}\right\}$. For each time point $t^{\prime}$ between subsequent considerations of items, grouping and classification remain unchanged. Formally, $\forall t^{\prime}=t+1, \ldots, n e x t(t, u)$ : $D_{u}^{t^{\prime}}=D_{u}^{\text {next }(t, u)} \wedge C_{u}^{t^{\prime}}=C_{u}^{\text {next }(t, u)} \wedge G_{u}^{t^{\prime}}=G_{u}^{\text {next }(t, u)}$.

\subsection{Initial classifier learning}

The task is to classify an item according to a set of concepts, based on its contents. We regard a classifier as an explanation why an object is assigned to a certain concept, i.e. its intensional definition. As indicated above, the opposite approach is the extensional definition, obtained by listing all objects that fall under that definition, and thus belong to the set or class for which the definition needs to be formed.

The goal is to determine intensional definitions for the user-generated groupings. Each cluster or group is then regarded as a class for which a definition needs to be calculated. Since different algorithms can be used for clustering, there are different ways in which these definitions can be calculated. These intensional definitions can then be used to assign new items to these clusters or groups. This can be seen as a classification task, since new, unclassified items need to be categorised.

\subsection{Choosing the classifier(s)}

The selection of peer guides whose classifiers are used in grouping requires a measure of user similarity or divergence. Each user organises a set of items which he is interested in. Multiple peer users organise different, possibly overlapping, subsets of $D$. We start from the idea that similarity of items in peers' groupings indicates their interest similarity. The question that arises is: how to define similarity/divergence between groupings of non-identical item sets? Using a measure based on the overlap of items, such as Jaccard index, or mutual information would fit as the similarity/divergence measure if item sets overlap to a large extent. However, it is usually the case that peers have access (or interest) to a limited number of items, and only a small number of popular (easily accessed) items are available to all peers. To overcome this, we define a new measure of divergence between peers.

We assume that a user groups items based on their features, e.g. text for documents. For users $u$ and $v$ and their respective groupings $G_{u}^{t}$ and $G_{v}^{t}$, we define the inter-guide measure of diversity udiv as: 


$$
\begin{aligned}
u \operatorname{div}(u, v)= & \frac{1}{2}\left(1 /\left|G_{u}^{t}\right| \sum_{x \in G_{u}^{t}} \min _{y \in G_{v}^{t}} \operatorname{gdiv}(x, y)\right. \\
& \left.+1 /\left|G_{v}^{t}\right| \sum_{y \in G_{v}^{t}} \min _{x \in G_{u}^{t}} \operatorname{gdiv}(y, x)\right)
\end{aligned}
$$

where $\operatorname{gdiv}(x, y)$, or inter-group diversity, is any divergence measure defined on two groups. ${ }^{1}$

The measure defined in Eq. 1 captures the differences between groupings by rewarding groups having shared items and groups containing similar items. The double average creates a symmetric measure.

We calculate the inter-guide divergence for all pairs of users $u, v \in U$ and create a $|U| \times|U|$ matrix $M_{\text {sim }}$. The $M_{\text {sim }}$ matrix is used to select the most similar users to the user who is structuring items. For each user, we extract the corresponding row from $M_{\text {sim }}$ and sort it to find the most similar peers, defined as the least divergent ones. From this list, we select the top $n$ users as guide(s).

\subsection{Classification}

In the classification step, the identified determining classifiers of the peer users from the previous step are now used to classify the item under consideration. We distinguish two cases: in the first one, the user guides himself, so the intensional model of his own structuring is used as classifier for the new item. In the other case, the user is guided by his peers.

Self-guided classification If the user has not seen the item yet, we can use the intensional description of the user's current clustering to classify the new ${ }^{2}$ instance.

At time $t$, the time where the new item arrives, the user's own classifier is applied: $x=C S_{u}^{t}(d)$. This gives the proposed group $x$ based on the intensional model of the user under consideration. The item $d$ is added to this $x$, which results in the new grouping $G S_{u}^{\text {next }(t, u)}$.

This is illustrated in Figure 1, which shows the self-guided classification of a user $u$. It starts from the observed grouping at time point $0, O G_{u}^{0}$, which is learned via classifier learning from the extensional definitions, i.e. the original

\footnotetext{
${ }^{1}$ If desired, a similarity measure can be calculated from this easily by appropriate normalisation: $\operatorname{usim}(u, v)=\left(\max _{w, z \in U} u \operatorname{div}(w, z)-u \operatorname{div}(u, v)\right) /\left(\max _{w, z \in U} u \operatorname{div}(w, z)\right.$.

${ }^{2}$ Note that there is another possible case, namely when an item arrives that a user has already seen. However, we were able to disregard this case given the nature of our data. We used CiteULike data, which is a social bookmarking system for scientific papers, where we determined the groupings based on the taggings. As already shown in [26], only a very small proportion of the taggings are produced by the user who originally introduced the item to the system, and in general, users do not add new tags to describe the items they collected and annotated once.
} 
grouping of user $u$. At each time step, when an item arrives, the previous intensional definition, i.e. classifier, from user $u$ is used to classify the new item. After the item is added, a new intensional definition is learned. For example, when item $b$ arrives, $C S_{u}^{1}$ is used to classify it, and a new classifier $C S_{u}^{2}$ is learned.

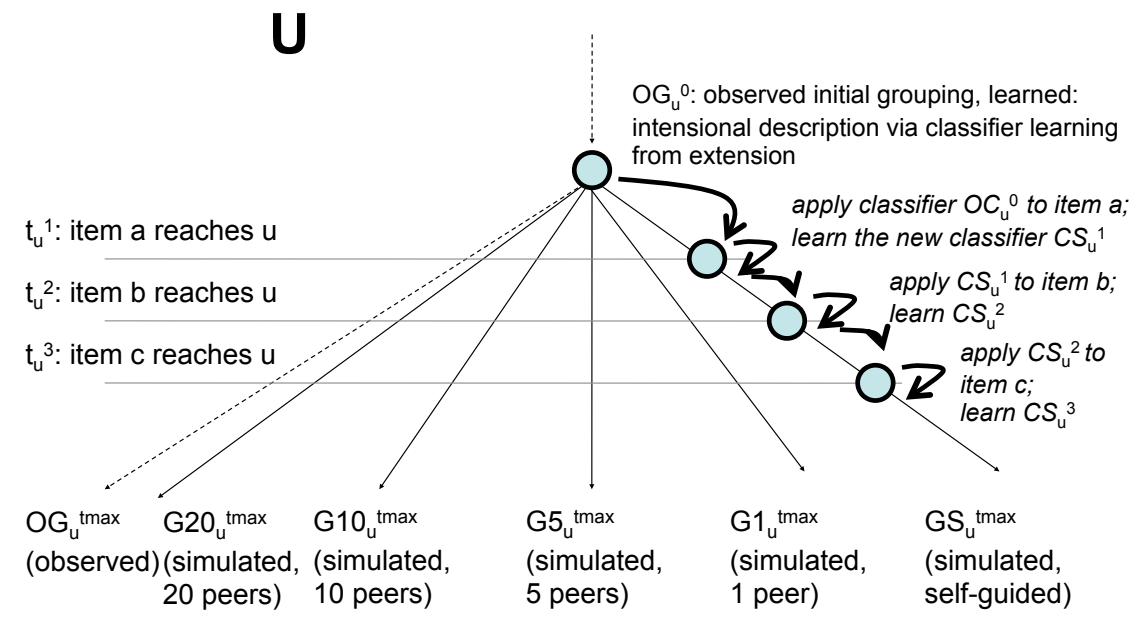

Fig. 1. General workflow for self-guided classification.

Peer-guided classification An alternative to self-guided grouping is to use the intensional descriptions of peer users to classify the new item. We distinguish two cases: guidance by the single most similar user, and guidance by the top $\mathrm{k}$ peers.

Top-1 peer If the peer user has already seen the item $d$, she has grouped it into one of her own groups; call this $y$. If she has not seen the item yet, she would classify it into her present structure into some $C 1_{u}^{t}(d)$; call this $y$ too. (Recall that the classifier is named $C 1_{u}^{t}$ because the peer is $u$ 's top- 1 peer at the current time.) This structuring is now projected back onto $u$ : he will group this item into that of his own groups that is most similar to $y$, or least dissimilar from $y$, namely into his group $x=\operatorname{argmin}_{x \in G 1_{u}^{t}} g \operatorname{div}(x, y)$. In both cases, $d$ is added to the resulting $x$. The new grouping is represented by $G 1_{u}^{\text {next }(t, u)}$.

This is illustrated in Figure 2, which shows the top-1 peer-guided classification of a user $u$, with peers $v$ and $w$. It starts from the observed grouping at time point $0, O G_{u}^{0}$, which is learned via classifier learning from the extensional definitions, i.e. the original groupings of user $u$. At each time step, when an item arrives, the most similar user is determined, and her intensional definition is used to classify the new item. After the item is added, a new intensional definition is learned. E.g. at the arrival of item $h$, user $v$ is identified as the most similar peer of user $u$, so consequently $C S_{v}^{1}$ is used to classify item $h$. 
More than 1 peer Let $v_{1}, \ldots, v_{k}$ be the top peers of $u$ at $t$. For each $v$, a clustering of user $u$ 's grouping is calculated, with the method described for the top-1 peer. Then $y=C S_{v}^{t}(d)$ is some $y \in G S_{v}^{t}$ ( $v$ 's group into which the item would be put). The result is a set of groupings, where majority voting decides on the final user group to put the item in. In case of ties, the largest $x \in G n_{u}^{t}$ is chosen.

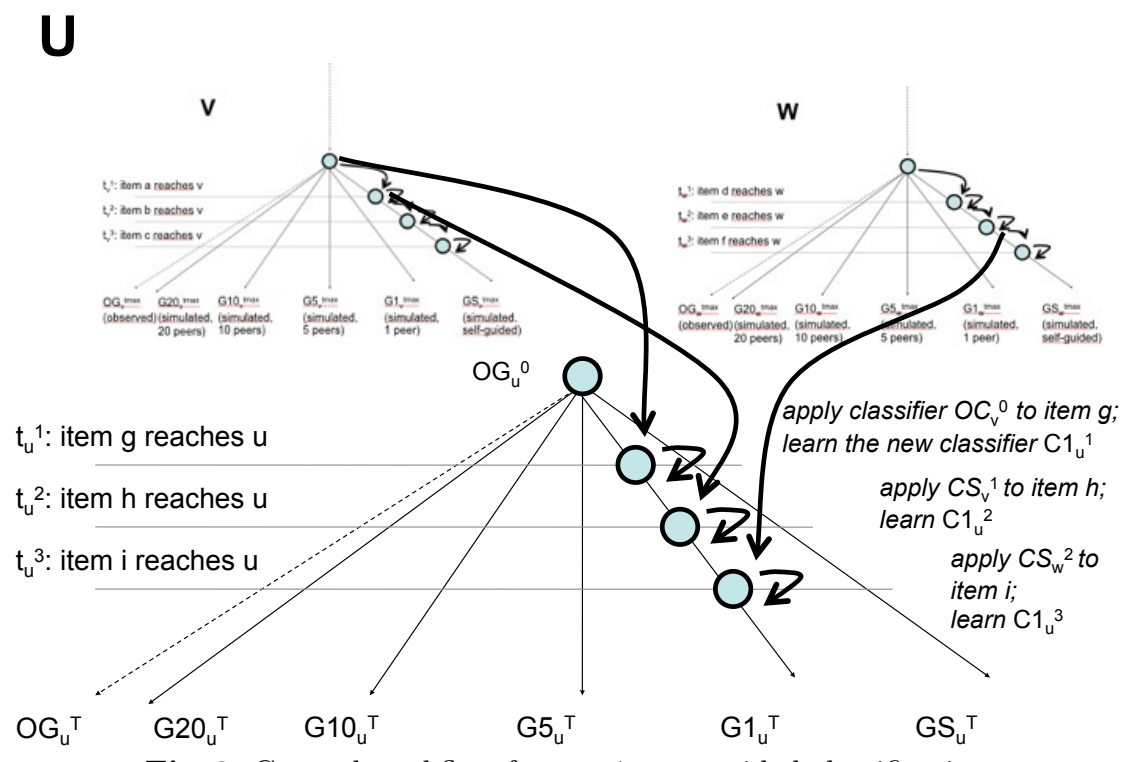

Fig. 2. General workflow for top-1 peer-guided classification.

\subsection{Intension update}

After the addition of the newly classified item, the intensional definition needs to be updated, which is needed to reflect the changed grouping structure implied by this addition. This is done by updating the classifier, which results in the new intensional definitions $C S_{u}^{n e x t(t, u)}, C 1_{u}^{n \operatorname{ext}(t, u)}$ and $C n_{u}^{n e x t(t, u)}$ for the self-guided, top-1 and top-n peer-guided classification respectively.

\section{Empirical results}

For testing the method outlined in the previous sections we ran a series of empirical analyses on CiteULike ${ }^{3}$, a social bookmarking system designed for scientific publications. A social bookmarking system is a perfect candidate for our grouping approach. Its Web 2.0 nature implies collaboration between peer users for enhancing the system, and by tagging, users implicitly group items. This was further motivated by the results of [26], which indicate that a rather low level of

\footnotetext{
$\overline{3}$ http://www.citeulike.org
} 
item re-tagging and tag reuse, together with the much larger number of items than tags in CiteULike, suggests that users exploit tags as an instrument to categorise items according to topics of interest. This is also indicated by results on usage patterns of collaborative tagging systems in [13]. Furthermore, they also indicate that the relatively high level of tag reuse suggests that users may have common interest over some topics. This motivated us to test whether this collaborative power and shared interests are the basis for users' organisations of documents, or whether they rather "trust" their own experience and are not "guided" by their peers.

In a social bookmarking system users apply free-text keywords (tags) to describe an item. One application of a tag by a user to a item is referred to as tagging. Combining multiple taggings by multiple users gives rise to a data structure that has been termed folksonomy [21]. A folksonomy can be viewed as a tripartite graph where nodes belong to sets of users, tags, or items and a hyper-edge is created between between a user, tag, and item for each tagging.

\subsection{Dataset}

We obtained a dump of the CiteULike database containing all entries from December 2004 to February 2010. In total, there were 486,250 unique tags, 70,230 users, 2,356,013 documents, and 10,236,568 taggings. As noted by previous work [13], the nodes and hyper-edges in a folksonomy have a long tail distribution, and most users tag a small number of items, and most items are tagged rarely. To overcome this sparsity, we followed a widely used folksonomy sampling method based on p-core subgraphs. The p-core subgraphs of a graph are its connected components in which the degree of each node must be at least equal to p. As in similar studies $[27,16]$, we set the value for $\mathrm{p}$ to 5 . Further, we constrained the dataset with regard to time and analysed only the taggings from 01/2009 until 02/2010. In total this dataset had 12,982 tags, 377 users, 11,400 documents, and 124,976 taggings. We refer to this folksonomy as $F$. For each document in the p-cores, we obtained the text of the abstract available on the CiteULike website.

\subsection{Initial grouping}

There are structures where people group explicitly, such as mail folders, and others where people group implicitly. Tagging is an example of the latter. Since organising and structuring items is one of the largest incentives for using a social bookmarking system [28], we assume that the tagging assignments the user has at the start of the learning phase are a reasonable starting point to create the initial groupings. In accordance with Section 3.1, we use the word "observed" for these groupings, fully aware that these are generated by clustering, but we wanted to make the distinction based on the data which was used for grouping (observed data).

In order to learn those observed groupings, we split the dataset into two parts. The first part, containing the first 7 months of our dataset, is used for learning the initial groupings $G_{\bullet}^{0}$. On this part, we apply a clustering algorithm 
as in $[4,3]$. For a user $u$, we first restrict $F$ to contain only nodes connected to $u$. This gives rise to a graph $F^{u}$. We then project $F^{u}$ to a graph $S F^{u}$ in which nodes belong to documents from set $D_{u}^{0}$ and edges are created between these nodes when some tag is applied to both documents. The weight of the edges in $S F^{u}$ is equal to the number of tags that two documents share in $F^{u}$. We then applied a modularity clustering algorithm [33] to partition the $S F^{u}$ graph. Each partition is treated as one group in $G_{u}^{0}$. This is repeated for all $u \in U$ to obtain initial groupings of all users, and resulted in an average of 6.59 groups per user.

The next step is to learn the initial classifiers. For this purpose, we used the Naive Bayes Classifier implementation in the WEKA data mining toolkit [14]. We used the bag of words representation of each publication's abstract as features for classification. This motivates our choice for Naive Bayes, since it has the property of being particularly suited when the dimensionality of the inputs is high. We also used a kernel estimator for modelling the attributes, rather than a single normal distribution, since this resulted in a better classification accuracy. The kernel estimator uses one Gaussian per observed value in the training data, and the conditional probability output by a kernel estimator is the weighted combination of the conditional probabilities computed from each of its constituent Gaussians.

\subsection{Simulating groupings}

We represent groups belonging to a grouping using language models. At time $t$ for a user $u$ for every of his groups $x \in G_{u}^{t}$ we create a language model $\Theta_{x}$. To find the most similar peers to a user $u$ we calculate his inter-guide divergence (Eq. 1) to all users $v \in U$. In our experiment on social bookmarking, as the intergroup divergence (gdiv) we used Jensen-Shannon divergence (JS) [7]. For two language models $\Theta_{x}$ and $\Theta_{y}$ representing groups $x$ and $y$ belonging to groupings $G_{u}^{\bullet}$ and $G_{v}^{\bullet}, J S$ is defined as:

$$
J S\left(\Theta_{x}, \Theta_{y}\right)=\frac{1}{2} K L\left(\Theta_{x}, \Theta_{z}\right)+\frac{1}{2} K L\left(\Theta_{y}, \Theta_{z}\right),
$$

where the probability of every word in $\Theta_{z}$ is the average probability in $\Theta_{x}$ and $\Theta_{y} ; K L\left(\Theta_{\bullet}, \Theta_{*}\right)$ is Kullback-Leibler divergence between two language models.

\subsection{Results}

Once we obtained the groupings for $G S$ and $G\{1|5| 10 \mid 20\}$ of one user, we compared these groupings with his observed groupings at $t_{\max }$. Like the observed initial grouping of a user $u$, his observed final grouping at $t_{\max }$ is a structuring of all the documents he has considered at that time. Every simulation run (whether it be guided by self, the top-1 peer, or the top-k peers) also considers the same sequence of documents arriving for $u$. Therefore, all simulated groupings and the observed grouping of one user at $t_{\max }$ contain the same document set. To compare these groupings, we investigated the similarity between them. Since the groupings to compare contain the same set of documents, we can use normalised 


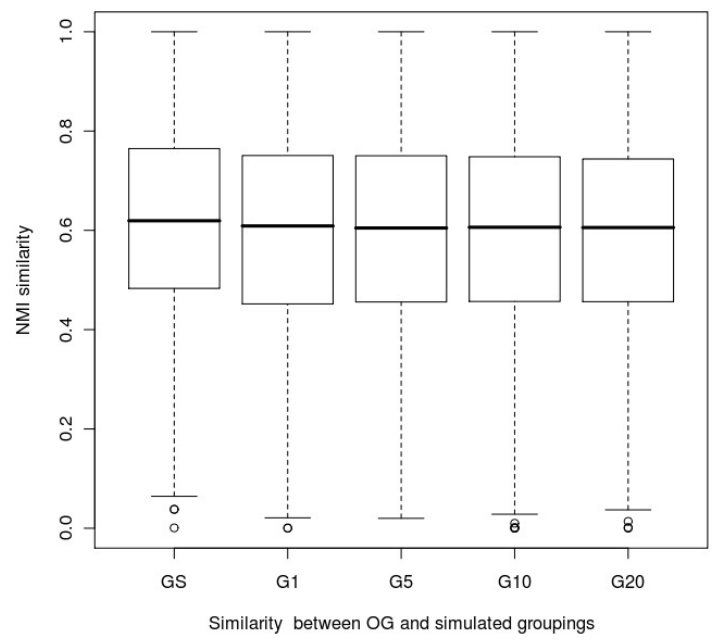

Fig. 3. Similarity between $O G$ and simulated groupings.

mutual information (NMI). It has desirable properties: it has a value between 0 and 1 , and it behaves well when calculated for groupings with different numbers of groups [24].

The normalised mutual information (specifically, NMI 4 [31]) of two groupings $G$ and $G^{\prime}$ is defined as

$$
N M I\left(G, G^{\prime}\right)=H(G)+H\left(G^{\prime}\right)-\frac{H\left(G, G^{\prime}\right)}{\sqrt{H(G) H\left(G^{\prime}\right)}}
$$

where $H(G)$ is the entropy of grouping $G$ and $H\left(G, G^{\prime}\right)$ the joint entropy of $G$ and $G^{\prime}$ together. ${ }^{4}$

The similarity results for all groupings are shown in Figure 3. Our research question was to investigate whether users are guided in structuring items by their own experience or by their peers. The results suggest that in spite of the collaborative nature of a social bookmarking system, users tend to keep to their structuring system. The NMI between $O G$ and $G S$ is highest having a mean of 0.61 (st.dev: 0.2). Using the closest peer grouping for grouping $(G 1)$ does not produce more similar groupings, having a mean of 0.57 (st.dev: 0.21 ). Including more guides into decision does not improve the results; in our experiment the best peer grouping is for $G 20$ (mean: 0.58, st.dev: 0.21). This is more similar to $O S$ when compared to $G 1$, but still less similar compared to $G S$.

Given the high standard deviation for all groupings, we investigated whether some users are more influenced by some guides. To discover this, we looked more closely into the distribution of NMI across all users. Figure 4 shows the results. We expected to see a more bimodal distribution if some users "prefer" different guides. However, all distributions fit to a normal distribution skewed to the

\footnotetext{
$\overline{4}$ for groups $x, y$ and $p$ the distribution of items over them:

$H(G)=-\sum_{x \in G} p(x) \log _{2} p(x)$ and $H\left(G, G^{\prime}\right)=-\sum_{x \in G} \sum_{y \in G^{\prime}} p(x, y) \log _{2} p(x, y)$.
} 

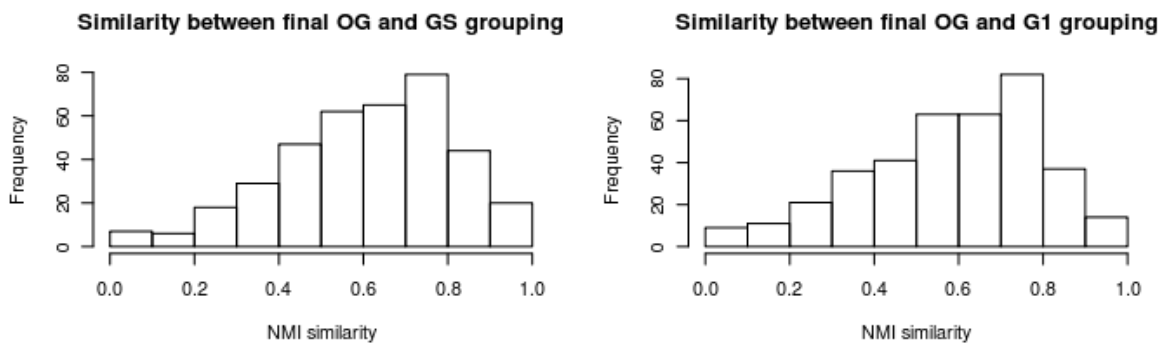

Similarity between final OG and G5 grouping

Similarity between final $O G$ and $G 10$ grouping
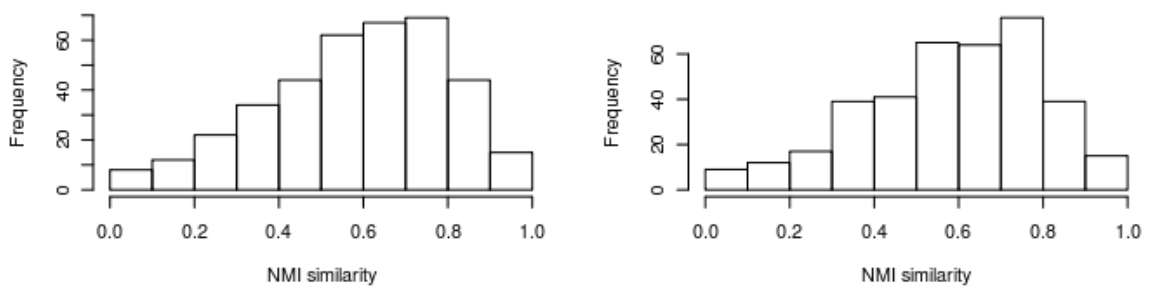

Similarity between final $O G$ and G20 grouping

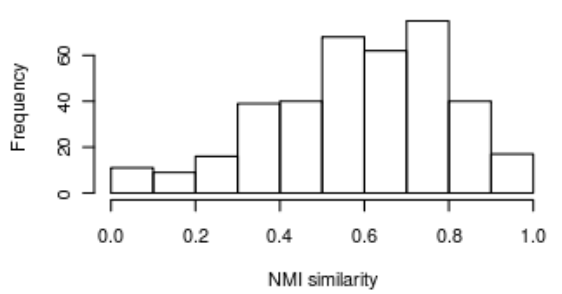

Fig. 4. Distribution of similarity between $O G$ and simulated groupings.

right (towards higher similarity). Normality of the distribution is tested using the Shapiro-Wilk test, and all p values are higher than 0.05 . This suggests that the majority of users behave in the same manner when it comes to structuring their items.

The results should be interpreted with some caution, and they lead to further research questions. The differences of fit were not very large and not significant. And a result that shows that on average, a user's observed grouping is 0.61 similar to his simulated self-guided result and 0.57 to the simulated peer-guided result, can mean two things: the simulated self-guided result and peer-guided result are quite similar, or they are quite different. We will explore this question further by considering our results in the light of related work.

Relation to other research. The results correspond to the results by Santos-Neto et al. [26] on individual and social behaviour in tagging system. The authors used a solution based on interest-sharing between users to infer an implicit social structure for the tagging community. They defined the interestsharing graph, where users are connected if they have tagged the same item or 
used the same tags and found out that users self-organise in three distinct regions: users with low activity and unique preferences for items and tags, users with high similarity among them, but isolated from the rest of the system, and a large number of users with mixed levels of interest sharing. The results are also compatible with the findings of [13], which - based on the usage patterns in collaborative tagging systems - indicates that users are drawn primarily to tagging systems by their personal content management needs, as opposed to the desire to collaborate with others. Our results extend those findings: Our approach not only looks at the tags they use, but also at the way they use the tags vis-à-vis one another, i.e. in their structuring. This can give an indication as to whether users are more self-guided than peer-guided.

The results of [22] may at first sight appear to point into a different direction: Musto et al. studied the accuracy of tag prediction based on self-guidance and community-guidance (in our terminology: all peers). They found that (a) the community-guided tag predictions were somewhat more accurate than the selfguided ones and that (b) a strategy that first extracts tag suggestions from the items themselves and then complements them with personal tags and then the community's tags produced the best accuracy. Closer inspection reveals, however, that the basic assumptions of that model and of our own form an interesting complementarity and may help to further interpret our results.

The basic difference is that we are not interested in the tags per se, but in the mental structure that they reveal. We will explain the complementarity using a very simple fictitious example. Assume a user $u$ and a user $v$ (who represents the whole community). $u$ has tagged items $D_{1}$ (some set of items) with the tag $t_{a g}$, and $D_{2}$ with $t_{a g} . v$ has tagged some $D_{3}$ with $t_{a g}$ and some $D_{4}$ with $\operatorname{tag}_{4}$. All 4 tags are different. A new item arrives for $u$ that is most similar to his group $D_{1}$ and to the community's group $D_{3}$. If the user is guided by self, our model will predict the new item to go into $D_{1}$; if he is peer-guided, our model will predict that the new item goes into that of u's groups that is most similar to $D_{3}$. Now assume that $D_{1}$ is most similar to $D_{3}$. This corresponds to a situation in which everybody more or less structures in the same way. Our model will then predict that the new item goes into $D_{1}$. And the final observed grouping will be quite similar to the self-guided simulated one, and also to the peer-guided simulated one. (And this is what we found.) Musto et al., in contrast, look at the tag manifestations of this mental structure. Their model predicts for selfguidance that the new item will be tagged with $\operatorname{tag}_{1}$, and for peer-guidance that it will be tagged with $\operatorname{tag}_{3}$. So if vocabulary converges in the population, the item will be tagged with $\operatorname{tag}_{1}$ and $\operatorname{tag}_{3}$, and the personal-plus-community model will produce the best prediction. (And this is what Musto et al. found.) Thus, one interpretation that is consistent with the results of both studies is that while there is some more self-guidance, the differences to peers are actually quite small, and individual vocabularies do tend to converge to the community's vocabulary.

This interpretation however relies on an assumption about dynamics (the convergence of vocabulary), and dynamics were not modelled by Musto et al. Also, there are combinations of equality/difference in grouping on the one hand 
and vocabulary on the other, that are associated with different predictions in the two models. These combinations may persist in specific subcommunities. Finally, the levels of accuracy/NMI that they/we measured indicate that other factors play an important role too in tagging. In future work, this should be investigated in more detail.

\section{Self-guided and peer-guided grouping: influence vs. interactive recommendations}

In the previous sections, we have investigated self-guided and peer-guided grouping as a model of how content organisation in social media may evolve. This is a study of influence as it (may have) happened. However, the grouping of new objects (and the continued learning of classifiers this entails) can also be used to recommend structure to a user. In other words, we can move from a descriptive study of influence to a prescriptive suggestion of influence. We have created systems embodying this idea in two domains: the structuring of scientific literature and "friends grouping" in online social networks.

\subsection{Interactive self-guided and peer-guided grouping: reference management}

The CiteSeerCluster and Damilicious tools [5,32] help users structure a list of scientific articles. Both assume that these lists are the results of using a search engine for a query $q$ for user $u$. This decision was made to allow us to integrate the tool with a real-world platform for literature search (CiteSeer), but it would be straightforward to also allow the user to upload a list obtained in some other way. To make the list more manageable, it is clustered into subgroups based on the textual contents or links to other articles, using typical clustering algorithms used and validated in bibliometrics. The user can then work with this structure, naming clusters based on proposals based on textual analysis, deleting or moving items from one cluster to another, or creating new clusters.

Each group of publications in the final grouping is regarded as the extensional definition of a concept. An intensional description of each concept is created by the top-10 TF.IDF terms in the grouped texts and, optionally, user input (CiteSeerCluster) respectively the top Lingo phrase of the group's original cluster (Damilicious). The advantage of the former is greater adaptivity, the advantage of the latter is better human understandability. Figure 5 (left) shows the Damilicious interface with group labels and publication IDs. (The latter can be clicked on for information and full-text access.) User $u$ may later re-use her own classification of the results to $q$ for a new query $q^{\prime}$, or she may use another user $v$ 's classification. This user $v$ may be selected on different grounds, and homophily is only one of them. Thus, a user may decide to not follow the groupings of the $v$ who is most similar to her, but to try and use the groupings least similar in order to broaden her horizon. 


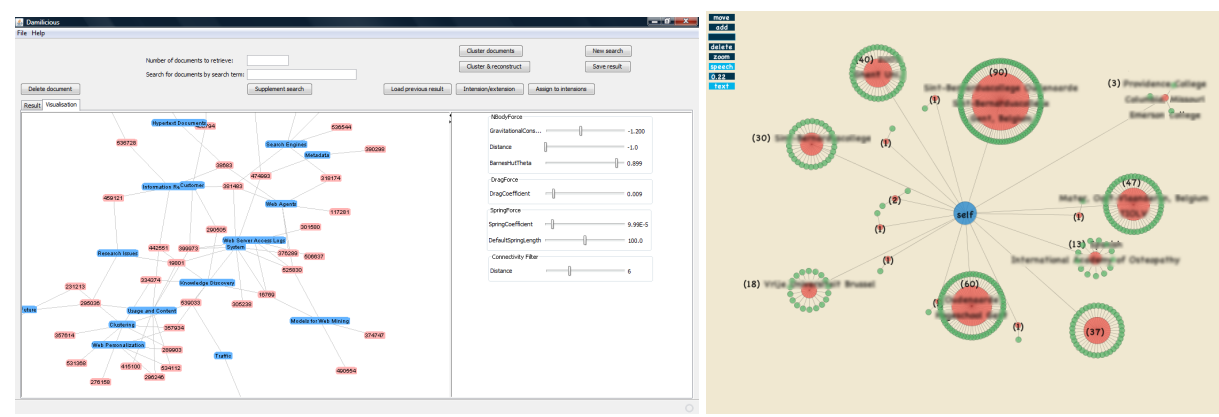

Fig. 5. Damilicious and FreeBu: groups and their elements as group-element graphs or as elements arranged "around" their groups

\subsection{Interactive self-guided and peer-guided grouping: online social networks}

The basic idea presented in the present paper can also be applied to other domains in which people categorise and influence one another in categorising. One domain that we are exploring in current work is that of "friend grouping" in online social networks.

In environments such as Facebook or LinkedIn, people assemble large collections of contacts - other users of the same online social network who want to come into or remain in contact with the present user, often for purposes of communicating with one another, but also to maintain weak social ties. The communication may also be unidirectional, as in the follows relationship in Twitter, where Twitter users that one "follows" often constitute one of many information sources. Sets of contacts grow quickly, and the average Facebook user now has several hundred "friends", with counts ranging from 141.5 overall to 510 as the value for the age group $18-24 .{ }^{5}$ Such unstructured multitudes may present problems for targeted communication and privacy management, since often messages are posted that should not really be read by everyone, and groups of contacts may be used as filters to selectively target certain recipients only. There may also be other reasons for bringing structure into sets of contacts, for example to make specific friends' activities more visible for oneself. ${ }^{6}$

Current commercial online social networks support such structuring in two forms: by allowing users to create groups of contacts manually (Google+ circles, Facebook lists) or by employing a simple-looking but undisclosed classification algorithm (Facebook smart lists appear to group by the values of attributes such as "school"). The idea of using more advanced forms of data mining for deriving

\footnotetext{
5 The first number results from dividing two numbers that Facebook reports in its 2013, first quarter, Financial results: the total number of friend connections divided by the total number of accounts ("users") [29]. The second number is the result of a 2000-people telephone survey [2].

${ }^{6}$ See for example Facebook's explanations of their lists, http://www.facebook.com/ help/204604196335128/.
} 
a good grouping structure has been pursued for a while $[9,17]$, and the search for the best algorithm to reconstruct "ground-truth" friend-grouping data is a topic of current research [19]. However, our recent findings indicate that the functionality currently offered is barely used, and it is also questionable whether a context-free type of grouping that works along the same criteria across different people actually exists.

Thus, learning such groupings for each user individually - and maybe also for uses in different contexts - appears to be a better approach. Also, since the machine learning can never be perfect, adding interactivity, i.e. the possibility to change the assignment of people to groups, suggests itself as useful. We have implemented this in our tool FreeBu [11], see Fig. 5 (right). It uses modularitybased clustering of the user's Facebook friend graph for the initial grouping, a choice motivated by a requirements analysis and validated by a comparison with other state-of-the-art algorithms on a ground-truth data set [12].

In an interview-based evaluation of the tool, we found that users were intrigued by the tool's functionality, and were curious as to how the groupings were derived. This calls for explanation options, and one way of explaining is an intensional description of the groupings. So far, the tool uses a characterisation ("labelling") in terms of labels derived from the grouped contacts' profiles. However, participants of our user study also remarked that this often leads to unintuitive results. The conceptual-clustering idea proposed in the present paper, in which the explanation is constructed from a classifier learned from the groups, is likely to be a better solution. We have tested this with a weightedkNN classifier and an explanation based on the group's extension plus common attributes of people in this group. We believe that such explanations functions will be key for empowering users through a better understanding of the offers of personalisation received from online social network platforms themselves or from add-on tools such as FreeBu.

So far, repeated (re-)groupings and learning have not been implemented as part of FreeBu, since we believe that a careful study of the usefulness and use of the created friends groups needs to precede such longer-term use cases, and since there are still many open questions regarding "friends management" in Facebook, the platform for which FreeBu is currently implemented. However, we expect that this will have a lot of potential, at least in online social networks that, like Twitter or LinkedIn, are treated in an "information-processing way" in which people may want group their contacts for example by information category, professional context, research area, or similar, and in which they may want an explicit and user-adaptive account of how they manage these categories.

Possible social effects in grouping friends, i.e. peer-guided decisions in grouping, are another area of future work for FreeBu. The whole idea of online social networks is built on "the social", but what does this mean? People are influenced by their friends in their choices of what to disclose and how to behave in online social networks. But (how) is the very fabric of the social network, namely the accepting and rejecting of friendship requests, and/or the mental model that people have of their social surroundings, shaped by peers and their 
influence? Future work could create the correlational and experimental settings in which these options are prescriptive suggestions, carry out user studies to evaluate them, and also investigate how datasets could be assembled in which such influences could be studied in descriptive settings.

\subsection{Evaluating interactive uses of grouping guides}

Interactive uses such as the ones described in the previous two sections require a very different form of evaluation than models of influence such as the one described in the main part of this paper. These evaluations cannot be performed on historical data, but must involve users. They also need to ask more questions beyond the focus of historical-data analysis, i.e. beyond "did this influence the current user's behaviour" (or in fact "is the current user's behaviour consistent with being influenced in this way"). First, the interactive tool should be one that people like using, so a number of measures of usability and perceived usefulness are an integral part. An example of this is shown in [5]. Second, the interactive tool should ideally produce something that is indeed useful for the user's current task - to measure this goal, one must also measure other criteria like "are the groups semantically meaningful in the application domain" [5] or "do the groups support another relevant behaviour in the application domain" [12].

The user-centric evaluations we performed $[5,12]$ suggest that the tools are a good support for their respective tasks and well-liked by users. Still, we believe that the tasks they "really" support (building up knowledge about scientific areas, communicating over a social-networking site) are more complex and longerterm than those that can be tested in the usual form of one-shot laboratory user studies, and that this should be investigated in future work [25].

\section{Summary and outlook}

Summary. In this research, we investigated collaborative grouping of items and built a framework that simulates this process. Specifically, we were interested in how different users structure items depending on the influence that guides this structuring process. We developed a new method that learns and combines classifiers for item set structuring. This method starts by, in the first step, learning a model for an existing grouping, which we referred to as the intensional definition. The second step uses these learned intensions to classify new items. To decide on the most appropriate users to take into account when grouping a new item, we defined a new divergence measure between groupings that may have different numbers and identities of elements. This method is applied to simulate the intellectual structuring process which underlies these structuring dynamics. We tested this approach on CiteULike, a social-bookmarking platform for literature management. The results of the study can have implications for system design of recommender systems and social search methods, for which a good understanding of the dynamics of these grouping activities is a prerequisite.

In addition to the simulation framework and its evaluation on historical data, we also described past and ongoing research on building interactive tools with 
which users can profit from their own and others' structuring of content and conceptual-clustering models learned from such structuring.

Limitations of the analysis of historical data. The main question we addressed was one of the difference in groupings, and we did not look at the benefits users have from adopting some guides. As a baseline we used observed groupings, which are not explicit user groupings, but implicit groupings learned based on users tag assignments. We are aware of the possible bias here and do not claim that these are the "best" groupings users can have. Also, we used a rather simple classifier, and a limited dataset. Our current analysis provides insights both into the grouping behaviour in general and into the behaviour of users in social bookmarking systems. In future work, with a more extensive dataset, this could be extended to an iterative approach where the groupings are evaluated at different time points to evaluate the impact of including new items in the construction of the classifiers.

Challenges for evaluation. The evaluation of grouping systems and therefore the design of better grouping systems face a number of challenges. One is the environment: If a recommender system operates in the platform itself (as is the case for example in Bibsonomy or CiteULike), then this may influence behaviour and therefore the historical data. Such influences are exacerbated in real-life applications such as reference management or social-network use, in which people also use other software such as search engines that also effectively act as recommenders. An interactive grouping system will also operate in such a pre-given environment and be affected by its recommender functionalities.

A special challenge for peer-guided grouping is privacy: an interactive grouping system divulges substantial information about the peer who is proposed as the "guide" or "donor" of a grouping. In contrast to recommender systems that recommend an item or a tag, the recommendation of a grouping will in many cases make it possible to identify the guide. This linking to a person may be a desired feature for the recipient user ("I want to group like someone who is knowledgeable"), but it may not be desired in all settings by the donor users. Thus, platforms offering peer-guided groupings should clearly describe this functionality and/or limit it to smaller groups whose participants trust each other and agree to this use of their data. These privacy concerns on the user side of course also imply privacy concerns on the data-controller side, which makes it more difficult for them to release historical datasets and for researchers to obtain such datasets for analysis.

Future work. Our method can be of use in different applications for (tag) recommendation and social search, where the grouping dynamics and behaviour adds a new level to the current individual and social measures used by these systems. Furthermore, we could extend the method to rearranging own groupings, based on the groupings of one's peers. The proposed method can also be combined with other metrics to create a hybrid measure for item set structuring.

In interactive applications, peers can be selected in different ways. One is to base the peer search on a relatedness based on personal acquaintance (as in social search), or on common properties, preferences, or behaviour (as in collab- 
orative filtering). Alternatively, relatedness can be based on different thinking. We believe that the latter, i.e. our approach to recommending structuring, can alleviate some filter-bubble side effects on relying only on a social network and instead leverage the diversity of internet users. For example, why should the same people (= one's set of contacts in a social network) be equally well equipped to give recommendations in totally different tasks? Finding guides based on the intellectual structure of a given task and its contents allows more flexibility and therefore potentially more quality.

Conclusion. We presented a study into grouping behaviour of users. Our framework combines different data mining methods to simulate collaborative grouping of items. The results of our experiment suggest that even in such open systems as social bookmarking tools, people tend to "trust" their own experience more than turn to the wisdom of the crowd. The main question we wish to follow in the future is not one of trust, but one of the "benefit" users get by being able to choose from a myriad of diverse groupings of the items they are interested in.

\section{References}

1. G. Adomavicius and A. Tuzhilin. Toward the next generation of recommender systems: a survey of the state-of-the-art and possible extensions. Knowledge and Data Engineering, IEEE Transactions on, 17(6):734-749, 2005.

2. Arbitron and Edison Research. The infinite dial 2013. navigating digital platforms. http://www. edisonresearch.com/wp-content/uploads/2013/04/ Edison_Research_Arbitron_Infinite_Dial_2013.pdf, May 2013.

3. Korinna Bade and Andreas Nürnberger. Creating a cluster hierarchy under constraints of a partially known hierarchy. In SDM, pages 13-24, 2008.

4. G. Begelman, P. Keller, and F. Smadja. Automated tag clustering: Improving search and exploration in the tag space. In Proceedings of the $W W W$ Collaborative Web Tagging Workshop, Edinburgh, Scotland, 2006.

5. B. Berendt, B. Krause, and S. Kolbe-Nusser. Intelligent scientific authoring tools: Interactive data mining for constructive uses of citation networks. Inf. Process. Manage., 46(1):1-10, January 2010.

6. Hendrik Blockeel, Luc De Raedt, and Jan Ramon. Top-down induction of clustering trees. In Proceedings of the Fifteenth International Conference on Machine Learning, ICML '98, pages 55-63, San Francisco, CA, USA, 1998. Morgan Kaufmann Publishers Inc.

7. Jop Briët and Peter Harremoës. Properties of classical and quantum jensenshannon divergence. Phys. Rev. A, 79:052311, May 2009.

8. David Carmel, Naama Zwerdling, Ido Guy, Shila Ofek-Koifman, Nadav Har'el, Inbal Ronen, Erel Uziel, Sivan Yogev, and Sergey Chernov. Personalized social search based on the user's social network. In Proceedings of the 18th ACM conference on Information and knowledge management, CIKM '09, pages 1227-1236, New York, NY, USA, 2009. ACM.

9. George Danezis. Inferring privacy policies for social networking services. In Proceedings of the 2nd ACM workshop on Security and artificial intelligence, AISec '09, pages 5-10, New York, NY, USA, 2009. ACM.

10. Brynn M. Evans and Ed H. Chi. Towards a model of understanding social search. In Proceedings of the 2008 ACM conference on Computer supported cooperative work, CSCW '08, pages 485-494, New York, NY, USA, 2008. ACM. 
11. Bo Gao, B. Berendt, D. Clarke, R. de Wolf, T. Peetz, J. Pierson, and R. Sayaf. Interactive grouping of friends in osn: Towards online context management. In Data Mining Workshops (ICDMW), 2012 IEEE 12th International Conference on, pages 555-562, 2012.

12. Bo Gao and Bettina Berendt. Circles, posts and privacy in egocentric social networks: An exploratory visualization approach. In Proceedings of the 2013 IEEE/ACM International Conference on Advances in Social Networks Analysis and Mining, ASONAM'13, New York, NY, USA, To appear. ACM.

13. Scott A. Golder and Bernardo A. Huberman. Usage patterns of collaborative tagging systems. J. Inf. Sci., 32(2):198-208, April 2006.

14. Mark Hall, Eibe Frank, Geoffrey Holmes, Bernhard Pfahringer, Peter Reutemann, and Ian H. Witten. The weka data mining software: an update. SIGKDD Explor. Newsl., 11(1):10-18, November 2009.

15. Jonathan L. Herlocker, Joseph A. Konstan, Loren G. Terveen, and John T. Riedl. Evaluating collaborative filtering recommender systems. ACM Trans. Inf. Syst., 22(1):5-53, January 2004.

16. Robert Jäschke, Leandro Marinho, Andreas Hotho, Lars Schmidt-Thieme, and Gerd Stumme. Tag recommendations in folksonomies. In Proceedings of the 11th European conference on Principles and Practice of Knowledge Discovery in Databases, PKDD 2007, pages 506-514, Berlin, Heidelberg, 2007. Springer-Verlag.

17. Simon Jones and Eamonn O'Neill. Feasibility of structural network clustering for group-based privacy control in social networks. In Proceedings of the Sixth Symposium on Usable Privacy and Security, SOUPS '10, pages 9:1-9:13, New York, NY, USA, 2010. ACM.

18. Vipul Kashyap and Amit Sheth. Semantic Heterogeneity in Global Information Systems: The Role of Metadata, Context and Ontologies. In Michael P. Papazoglou and Gunter Schlageter, editors, Cooperative Information Systems, pages 139-178. Academic Press, San Diego, 1998.

19. Julian J. McAuley and Jure Leskovec. Learning to discover social circles in ego networks. In NIPS, pages 548-556, 2012.

20. Ryszard S. Michalski and Robert E. Stepp. Learning from observation: Conceptual clustering. In Ryszard S. Michalski, Jaime G. Carbonell, and Tom M. Mitchell, editors, Machine Learning: An Artificial Intelligence Approach, chapter 11, pages 331-364. Tioga, 1983.

21. Peter Mika. Ontologies are us: A unified model of social networks and semantics. Web Semant., 5(1):5-15, March 2007.

22. Cataldo Musto, Fedelucio Narducci, Pasquale Lops, and Marco Gemmis. Combining collaborative and content-based techniques for tag recommendation. In Francesco Buccafurri and Giovanni Semeraro, editors, E-Commerce and Web Technologies, volume 61 of Lecture Notes in Business Information Processing, pages 13-23. Springer Berlin Heidelberg, 2010.

23. Andreas Nrnberger and Sebastian Stober. User modelling for interactive useradaptive collection structuring. In Nozha Boujemaa, Marcin Detyniecki, and Andreas Nrnberger, editors, Adaptive Multimedia Retrieval: Retrieval, User, and Semantics, volume 4918 of Lecture Notes in Computer Science, pages 95-108. Springer Berlin Heidelberg, 2008.

24. Darius Pfitzner, Richard Leibbrandt, and David Powers. Characterization and evaluation of similarity measures for pairs of clusterings. Knowl. Inf. Syst., 19(3):361394, May 2009 
25. Catherine Plaisant. The challenge of information visualization evaluation. In Proceedings of the working conference on Advanced visual interfaces, AVI '04, pages 109-116, New York, NY, USA, 2004. ACM.

26. Elizeu Santos-Neto, David Condon, Nazareno Andrade, Adriana Iamnitchi, and Matei Ripeanu. Individual and social behavior in tagging systems. In Proceedings of the 20th ACM conference on Hypertext and hypermedia, HT '09, pages 183-192, New York, NY, USA, 2009. ACM.

27. Christoph Schmitz, Andreas Hotho, Robert Jschke, and Gerd Stumme. Mining association rules in folksonomies. In Vladimir Batagelj, Hans-Hermann Bock, Anuka Ferligoj, and Ale iberna, editors, Data Science and Classification, Studies in Classification, Data Analysis, and Knowledge Organization, pages 261-270. Springer Berlin Heidelberg, 2006.

28. Shilad Sen, Shyong K. Lam, Al Mamunur Rashid, Dan Cosley, Dan Frankowski, Jeremy Osterhouse, F. Maxwell Harper, and John Riedl. tagging, communities, vocabulary, evolution. In Proceedings of the 2006 20th anniversary conference on Computer supported cooperative work, CSCW '06, pages 181-190, New York, NY, USA, 2006. ACM.

29. Craig Smith. By the numbers: 32 amazing facebook stats. http://expandedramblings.com/index.php/ by-the-numbers-17-amazing-facebook-stats/, May 2013.

30. Rodolfo Stecher, Claudia Niedere, Wolfgang Nejdl, and Paolo Bouquet. Adaptive ontology re-use: finding and re-using sub-ontologies. IJWIS, 4(2):198-214, 2008.

31. Alexander Strehl and Joydeep Ghosh. Cluster ensembles - a knowledge reuse framework for combining multiple partitions. J. Mach. Learn. Res., 3:583-617, March 2003.

32. Mathias Verbeke, Bettina Berendt, and Siegfried Nijssen. Data mining, interactive semantic structuring, and collaboration: a diversity-aware method for sense-making in search. In Claudia Niederee, editor, Proceedings of First International Workshop on Living Web, Collocated with the 8th International Semantic Web Conference (ISWC-2009), Washington D.C., USA, October 26, 2009,, page 8. CEUR-WS, October 2009.

33. Li Wan, Jianxin Liao, and Xiaomin Zhu. Cdpm: Finding and evaluating community structure in social networks. In Changjie Tang, CharlesX. Ling, Xiaofang Zhou, NickJ. Cercone, and Xue Li, editors, Advanced Data Mining and Applications, volume 5139 of Lecture Notes in Computer Science, pages 620-627. Springer Berlin Heidelberg, 2008.

34. Bernard enko, Sao Deroski, and Jan Struyf. Learning predictive clustering rules. In Francesco Bonchi and Jean-Franois Boulicaut, editors, Knowledge Discovery in Inductive Databases, volume 3933 of Lecture Notes in Computer Science, pages 234-250. Springer Berlin Heidelberg, 2006. 